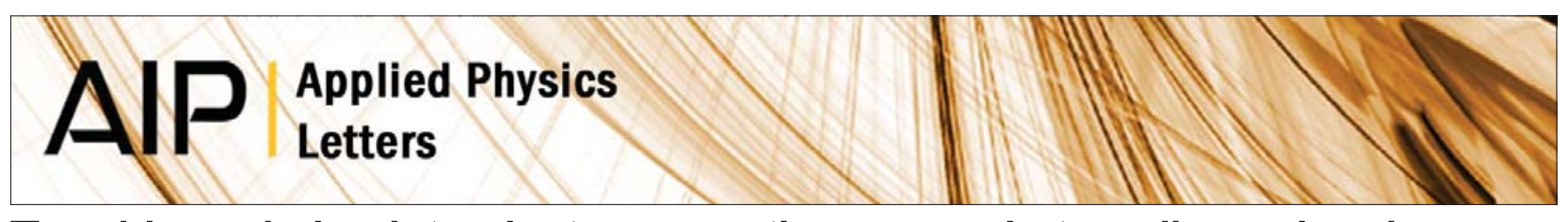

\title{
Tunable and absolute electromagnetic vacuum in two-dimensional photonic-band-gap Based on multiferroic materials
}

Kai Chen, Wei Jia, Yanbin Chen, Minghui Lu, Xirui Zhang et al.

Citation: Appl. Phys. Lett. 99, 132903 (2011); doi: 10.1063/1.3643053

View online: http://dx.doi.org/10.1063/1.3643053

View Table of Contents: http://apl.aip.org/resource/1/APPLAB/v99/i13

Published by the American Institute of Physics.

\section{Related Articles}

Superprism phenomenon in two-dimensional magnetophotonic crystals: Experiment and numerical simulation J. Appl. Phys. 111, 07E508 (2012)

Multicolor Čerenkov conical beams generation by cascaded- $\chi(2)$ processes in radially poled nonlinear photonic crystals

Appl. Phys. Lett. 100, 101101 (2012)

Improved fake mode free plane-wave expansion and three planar-slab waveguides method J. Appl. Phys. 111, 053103 (2012)

Electric-field-tuned color in photonic crystal elastomers Appl. Phys. Lett. 100, 101902 (2012)

High-Q aluminum nitride photonic crystal nanobeam cavities Appl. Phys. Lett. 100, 091105 (2012)

\section{Additional information on Appl. Phys. Lett.}

Journal Homepage: http://apl.aip.org/

Journal Information: http://apl.aip.org/about/about_the_journal

Top downloads: http://apl.aip.org/features/most_downloaded

Information for Authors: http://apl.aip.org/authors

\section{ADVERTISEMENT}

\section{NEW!}

iPeerReview

AlP's Newest App

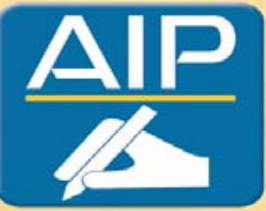

Authors...

Reviewers...

Check the status of

submitted papers remotely!

AIP Publishing 


\title{
Tunable and absolute electromagnetic vacuum in two-dimensional photonic-band-gap Based on multiferroic materials
}

\author{
Kai Chen, ${ }^{1, a)}$ Wei Jia, ${ }^{1}$ Yanbin Chen, ${ }^{2}$ Minghui Lu, ${ }^{2}$ Xirui Zhang, ${ }^{1}$ Wei Yang, ${ }^{1}$ Yong Wu, ${ }^{1}$ \\ Chenxi Huang, ${ }^{1}$ Xiangyin $\mathrm{Li},{ }^{1} \mathrm{Jie}$ Su, ${ }^{2}$ Junming Liu, ${ }^{2}$ Xiaomei Lu, ${ }^{2, a)}$ Jinsong Zhu, ${ }^{2}$ \\ and Yun Liu ${ }^{3}$ \\ ${ }^{1}$ Department of Physics, Nanjing University of Science and Technology, Nanjing 210094, \\ People's Republic of China \\ ${ }^{2}$ National Laboratory of Solid State Microstructures, Nanjing University, Nanjing 210093, \\ People's Republic of China \\ ${ }^{3}$ Research School of Chemistry Bldg 3, Australian National University, Canberra ACT 0200, Australia
}

(Received 31 March 2011; accepted 31 August 2011; published online 28 September 2011; publisher error corrected 30 September 2011)

\begin{abstract}
When multiferroic terbium manganite $\left(\mathrm{TbMnO}_{3}\right)$ crystal cylinders are periodically arranged in a square lattice, the resulting two-dimensional (2D) system exhibits photonic band gaps (PBGs). The absolute PBG originating from the Mie resonance is modulated from closed to open by applying an external static magnetic field, which is attributed to the electromagnon depression of the dielectric constant by the rearrangement of antiferromagnetic order. Tunable electromagnetic band structure may be realized by controlling the magnetic transition of manganese spins in $\mathrm{TbMnO}_{3}$. (C) 2011 American Institute of Physics. [doi:10.1063/1.3643053]
\end{abstract}

In the past years, the concepts of solid state physics have been brought to bear on electromagnetism and Maxwell's equations. In analogy to the case of electron waves propagating in a crystal, light waves traveling in artificially periodic dielectric structures have been described in terms of photonic bands with possible frequency gaps, where the propagation of electromagnetic (EM) waves is prohibited. There has been widespread interest in structural long-range order and Bloch waves for studying photonic band gaps in artificially periodic dielectric structures, especially after the pioneering works of Yablonovitch ${ }^{1}$ and $\mathrm{John}^{2}$ Intense effort has been made over the years to fabricate photonic band gap (PBG) materials. ${ }^{3,4}$ An effective way to modulate PBGs externally, i.e., tunability, was achieved with threedimensional PBG structures, which opened the door to tunable PBG materials. ${ }^{5}$ The extension of tunability to $2 \mathrm{D}$ PBG systems has been proposed. In those $2 \mathrm{D}$ cases, a clear tunable PBG is formed only for TM (or TE) modes and no "absolute" 2D PBGs (PBGs both for TM and TE modes) are realized. However, 2D PBG materials with absolute band gaps have several important uses, such as a feedback mirror in laser diodes.

For some applications, it is advantageous to obtain faster tunability of photonic band structure through an external static magnetic field (ESMF). Such tunability may be obtained by controlling magnetic orders. Multiferroic materials simultaneously possess ferroelectric, (anti)ferromagnetic, or ferroelastic orders in a single phase. ${ }^{6}$ For a long time, ferroelectricity and magnetism have been thought to be essentially independent or weakly interacting. Recently, however, a class of multiferroics, such as $\mathrm{TbMnO}_{3}$ (Ref. 7) and $\mathrm{RMn}_{2} \mathrm{O}_{5}(\mathrm{R}=\mathrm{Y} \text {, rare earth metal, } \mathrm{Bi})^{8}$ have been discovered, in which antiferromagnetic ordering itself is thought to induce ferroelectricity. Unlike "conventional" multiferroics, magnetoelectric coupling in these materials is very strong, leading to spectacular magnetodielectric effects, such as a change of dielectric constant at terahertz frequencies upon appli-

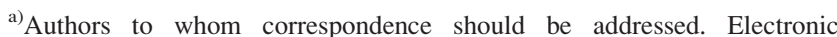
addresses: pacinonono@yahoo.com.cn and xiaomeil@nju.edu.cn.
}

cation of an external static magnetic field. ${ }^{9}$ The science of multiferroics has highlighted their application to higher-density solidstate memory devices. ${ }^{6}$ However, little attention has been paid to possible photonic phenomena, and only one work has reported enhancement of the magnetochiral effect using the broken symmetry of multiferroic crystallinenature. ${ }^{10}$ Multiferroic photonicband-gap materials, which are made of magnetodielectric-effect multiferroics periodically arranged in different backgrounds, have potential in next-generation optoelectronic devices.

In this letter, we propose forming a tunable and absolute PBG in a multiferroic photonic-band-gap material made of $\mathrm{TbMnO}_{3}$ crystals arranged in a square lattice. The $\boldsymbol{a}$-axis dielectric constant of $\mathrm{TbMnO}_{3}$ at terahertz frequencies is tuned by using the ESMF. The absolute gap opens significantly and can even be closed in such a 2D system.

Maxwell's equations for such a 2D structure can be decomposed into two equations satisfied by TE- and TM- modes as well as the photonic band structures. We did theoretical calculations for photonic structures with and without an ESMF using the finite difference time domain method with 289 plane waves. The convergence accuracy for several lowest photonic bands is better than $1 \%$. The angular frequency was normalized by the factor $a / 2 \pi c$ with $c$ the speed of light in vacuum. Our results show that TE- or TM-mode PBGs appear for absolute and tunable PBGs only below the normalized frequency 0.5 .

Figure 1 shows the magnetodielectric effect of a $\mathrm{TbMnO}_{3}$ crystal at the temperature 12 Kelvin $(\mathrm{K}) .{ }^{10}$ After applying the 8 Tesla (T) ESMF parallel to the $c$ axis of $\mathrm{TbMnO}_{3}$, the real part $\varepsilon^{\prime}$ and imaginary part $\varepsilon^{\prime \prime}$ of the $\boldsymbol{a}$-axis dielectric constant clearly change. This is due to the corresponding electromagnon change of the dielectric constant in the antiferromagnetic-order transition of $\mathrm{Tb}$ spins, which is from a modulated antiferromagnetic/ ferroelectric (AFM/FE) phase to a canted-antiferromagnetic (CA-AFM) phase, ${ }^{11}$ as shown in the inset. In our frequency range with a $24-\mathrm{GHz}$ width, the variation of the dielectric constant with the frequency is less than $1 \%$, so we assume its real and imaginary parts not to be dispersive under a ESMF. 


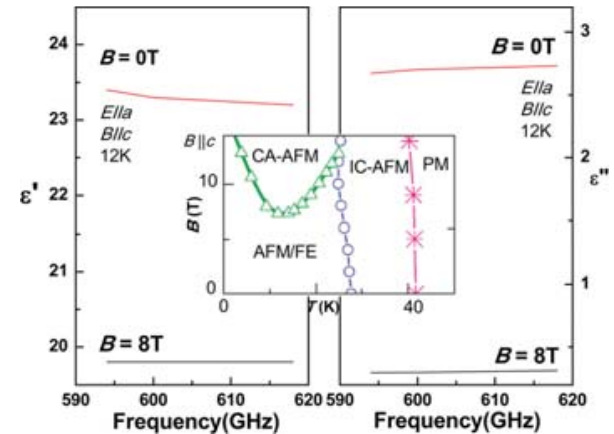

FIG. 1. (Color online) Terahertz-magnetodielectric effect in $\mathrm{TbMnO}_{3}$ crystal. Real part $\varepsilon^{\prime}$ and imaginary part $\varepsilon^{\prime \prime}$ of the $\boldsymbol{a}$-axis dielectric constant in $\mathrm{TbMnO}_{3}$ as a function of frequency. The external magnetic field is parallel to the $c$ axis and the ac electric field $(E)$ parallel to the $a$ axis. The insets show schematically the $(B, T)$ phase diagrams of the $\mathrm{TbMnO}_{3}$ crystal, where $B$ is the magnetic flux density of external static magnetic field and $T$ the temperature. Such magnetodielectric effect is the base of possible absolute and tunable gap in our $2 \mathrm{D}$ example.

The multiferroic photonic-band-gap material we propose is made of $\mathrm{TbMnO}_{3}$ crystal cylinders arranged in a square lattice (lattice parameter $a$ ) with air as the background, as shown in the inset of Fig. 2. For simplicity without losing generality, the PBG system's axis is parallel to the extension direction of the cylinders (the $c$ axis of $\mathrm{TbMnO}_{3}$ and the direction of the applied ESMF), and the EM waves propagate along the $b$ axis of $\mathrm{TbMnO}_{3}$. The TE-mode photonic band structure is shown in Figure 2, where the cylinder radius is $0.4 a$. When the ESMF is $0 \mathrm{~T}$, three PBGs are clear and their center frequencies are $0.155,0.270$, and 0.385 , respectively, as shown in the right panel of Fig. 2. We find that when the value of the lattice parameter $(a)$ is changed, the first PBG is centered at almost the same position and the photonic structures are nearly same, showing a scale-length invariant behavior. We, therefore, conclude that the first PBG is dominated by Bragg scattering. When an 8-T ESMF is applied (the left panel of Fig. 2), most smooth bands become pointed except the photonic structure around the first PBG. The center frequency of the first PBG is moved up by 0.01 and the $\mathrm{PBG}$ width is almost unaltered. All of this shows that the ESMF only has little effect on the first PBG and the photonic band structure nearby. Our results are in agreement with recent experimental and theoretical results that show a tiny shift in frequency of Bragg-dominating PBGs in response to an ESMF. ${ }^{12}$ The center frequency of the second $\mathrm{PBG}$ shows its relation to some electric modes of $\mathrm{TbMnO}_{3}$ crystals for EM absorption. ${ }^{9}$ The third PBG for absolute and tunable PBG is discussed below.

Figure 3 shows the corresponding TM-mode photonic band structure. Two gaps appear with center frequencies of 0.220 and 0.395. The first gap is rather wide (right panel of Fig. 3). By applying the 8-T ESMF, the first gap is reduced by $97.5 \%$ and even remains. This shows that the gap originates from antiferromagnetic resonances, i.e., magnetic modes, because the ESMF can only rearrange the antiferromagnetic order. ${ }^{13}$

To clarify the tunable and absolute gap, Fig. 4 shows TEand TM- mode photonic band structures without the ESMF, which constitute the fundamental result of this work. No bands intervene between the frequency 0.39 and 0.40 . The absolute gap appears with the moderate width $5 \%$ of the center frequency, which has contributions from the third TE-mode gap and the sec-

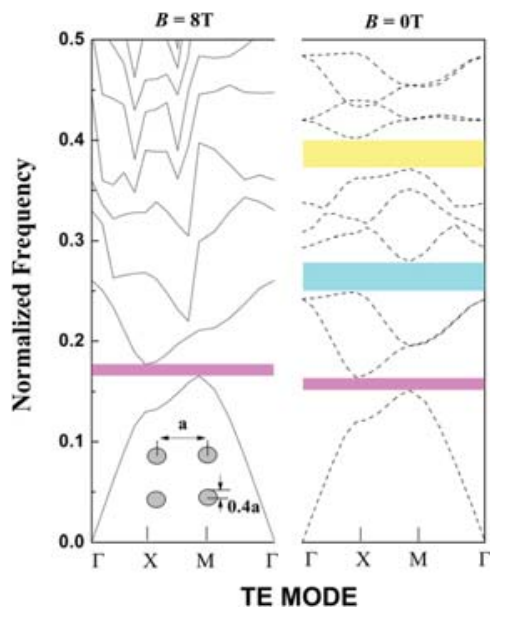

FIG. 2. (Color online) TE-mode photonic band structure along high symmetry lines $(\Gamma-\mathrm{X}-\mathrm{M}-\Gamma)$ in the Brillouin zone with an external static magnetic field of $8 / 0 \mathrm{~T}$. The inset shows the square lattice with the parameter $\sim a$. The third PBG has contributions to absolute and tunable PBG.

ond TM-mode gap. Despite the mode difference, the similar band shape nearby shows that the absolute PBG is dominated by the same resonance. Noticeably, the absolute PBG is closed by applying the ESMF, as shown in the left panel of Figs. 1 and 2. Because the ESMF moves the Bragg-dominating gap, we conclude that the absolute gap is not related to such large-scale geometric resonance. To highlight the possible resonance type, we present the EM intensity distribution of the PBG bottom or top at the high symmetry point $\Gamma$ in the upper inset of Fig. 4. Both TEand TM- mode EM waves are effectively confined within the cylinder. The intensity of EM waves at the gap top distributes as a circle, which has higher symmetry than the four-leaf-clover shape at the gap bottom. Also, the intensity was strongly enhanced in the center of the circle or 'leaf.' For an individual cylinder without the EMSF (the magnetic permeability defaulted as 1), the Mie resonance formulations for the scattered and internal fields subject to an incident plane wave are simplified as: ${ }^{14}$

for TE mode,

$$
H^{s c a t}=\sum_{-\infty}^{\infty} a_{n} H_{n}(k \rho) e^{-i n \phi}, \quad H^{\mathrm{int}}=\sum_{-\infty}^{\infty} b_{n} J_{n}\left(\sqrt{\varepsilon^{\prime}} k \rho e^{-i n \phi}\right)
$$

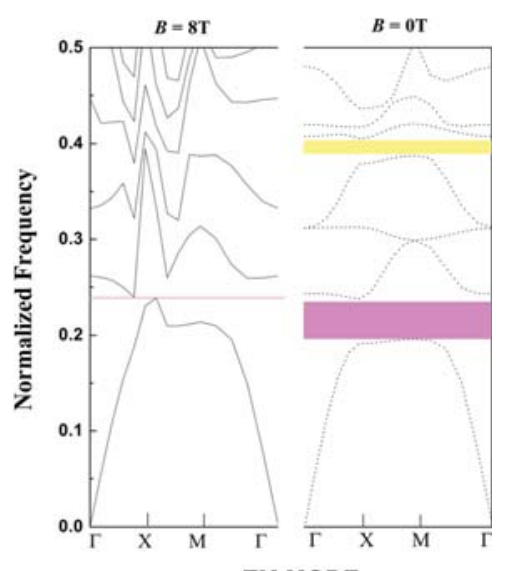

TM MODE

FIG. 3. (Color online) TM-mode photonic band structure. The second PBG appears for absolute and tunable PBG. 


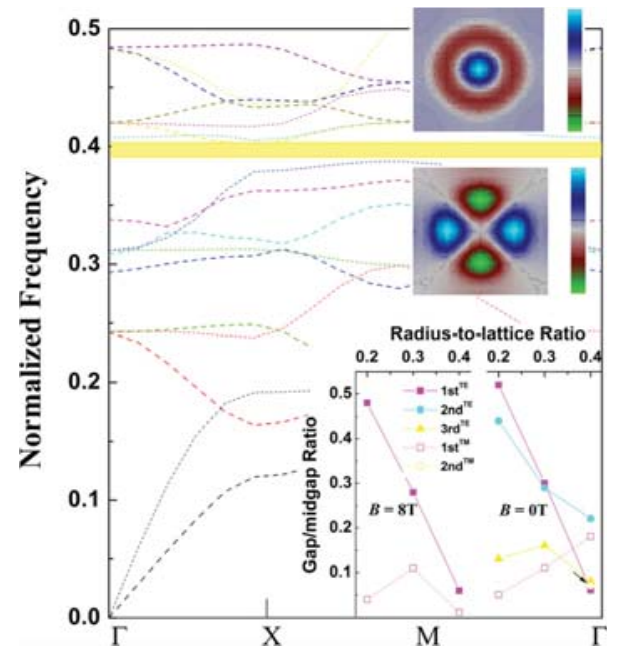

FIG. 4. (Color online) Absolute gap of the band structure without the external static magnetic field. The dashed lines are the TE-mode photonic band structure and the dotted lines are the TM-mode one. The upper insets show the electromagnetic intensity distribution of the gap bottom or top at the high-symmetry point $\Gamma$, which indicates that the absolute and tunable PBG is due to the Mie resonance. That is, the tunable and absolute gap is related to the EM absorption of a single cylinder of the $\mathrm{TbMnO}_{3}$ crystal itself. The lower inset shows the gap-to-midgap-frequency ratio as a function of radial contrast in an external static magnetic field of $8 / 0$ Tesla.

$$
\begin{aligned}
& a_{n}=i^{n} \frac{J_{n}(x) J_{n}^{\prime}\left(x_{1}\right)-\sqrt{\varepsilon} J_{n}^{\prime}(x) J_{n}\left(x_{1}\right)}{\sqrt{\varepsilon^{\prime}} H_{n}^{\prime}(x) J_{n}\left(x_{1}\right)-H_{n}(x) J_{n}^{\prime}\left(x_{1}\right)}, \\
& b_{n}=i^{n} \frac{2 i / \pi x}{\sqrt{\varepsilon^{\prime}} H_{n}^{\prime}(x) J_{n}\left(x_{1}\right)-H_{n}(x) J_{n}^{\prime}\left(x_{1}\right)},
\end{aligned}
$$

for TM mode,

$$
\begin{gathered}
E^{\text {scat }}=\sum_{-\infty}^{\infty} a_{n} H_{n}(k \rho) e^{-i n \phi}, \quad E^{\mathrm{int}}=\sum_{-\infty}^{\infty} b_{n} J_{n}\left(\sqrt{\varepsilon^{\prime}} k \rho e^{-i n \phi}\right), \\
a_{n}=i^{n} \frac{\sqrt{\varepsilon^{\prime}} J_{n}(x) J_{n}^{\prime}\left(x_{1}\right)-J_{n}^{\prime}(x) J_{n}\left(x_{1}\right)}{H_{n}^{\prime}(x) J_{n}\left(x_{1}\right)-\sqrt{\varepsilon^{\prime}} H_{n}(x) J_{n}^{\prime}\left(x_{1}\right)}, \\
b_{n}=i^{n} \frac{2 i / \pi x}{H_{n}^{\prime}(x) J_{n}\left(x_{1}\right)-\sqrt{\varepsilon^{\prime}} H_{n}(x) J_{n}^{\prime}\left(x_{1}\right)},
\end{gathered}
$$

where $\rho$ and $\phi$ are the cylindrical coordinates with the origin at the cylinder center, $x \equiv 0.4 a k=0.4 a \omega / c, x_{1}=\sqrt{\varepsilon^{\prime}} x, k$, and $\omega$ are the wave vector and the angular frequency, respectively, $J_{n}(x)$ and $H_{n}(x)$ are the nth order Bessel function and Hankel function of the first kind, respectively, and the prime denotes a derivative with respect to the argument. The Mie resonance of these gaps corresponds to divergence of the amplitude coefficients for the scattered and internal fields, i.e., the vanishing denominator of $a_{n}$ or $b_{n}$. The intensity distributions in the inset of Fig. 4 are the same as those features of a cylinder described by the Mie resonance formulation. Therefore, all of this shows that the tunable and absolute gap originates from the first or highorder Mie resonance. That is, the tunable and absolute gap is related to the EM absorption of a single cylinder of the $\mathrm{TbMnO}_{3}$ crystal itself. ${ }^{15,16}$ The manganese spins of $\mathrm{TbMnO}_{3}$ at $12 \mathrm{~K}$ without the ESMF are in a modulated AFM/FE phase. The refractive index of the $\mathrm{TbMnO}_{3}$ cylinders, $\sim 4.9$, is larger than that of the surrounding air. Thus, $\mathrm{TbMnO}_{3}$ cylinders constitute a se- ries of potential barriers to prohibit the propagation of EM waves, corresponding to strongly localized photon states. That is, the photonic states are spatially inside the cylinders and fully inhibited; other photons of transporting EM waves cannot take up these energy states, and propagation of EM waves inside the cylinder is forbidden and located. Pimenov et al. have shown that an 8-T ESMF can induce a transition of Tb spins to a CA$\mathrm{AFM}$ phase at $12 \mathrm{~K}$, and the antiferromagnetic order is rearranged and the magnetic permeability modulated. More importantly, the electromagnons' contribution to the $a$-axis dielectric constant can be suppressed during the magnetic structure transition, and as a result, the imaginary part of the $a$-axis dielectric constant is decreased by more than a factor of two and the real part is reduced by about 2 . $^{9}$ Therefore, the absorption of cylinder as an attractive potential decreases, and so does the potential barrier surrounding the cylinder. It is then possible for the third TEmode gap to close. At the same time, the absolute PBG is turned off. The lower inset of Figure 4 schematically shows the photonic band structure with different radius-to-lattice ratios. The absolute and tunable gap opens only in cylinders with $0.4 a$ radius, as the arrow indicates. Despite different cylindrical radii, the ESMF has almost the same effect on the first TE-mode PBG.

In summary, we investigated the possibility of a tunable and absolute PBG in a 2D square-lattice structure using the magnetodielectric effect of magnetic transition in a multiferroic $\mathrm{TbMnO}_{3}$ crystal.

The authors are grateful for financial support from the Outstanding Foundation of NJUST, the NJUST Research Funding (No. 2010ZDJH06), the National Natural Science Foundation of China (Grant Nos. 11004106, 50672034, 50832002, and 50901042), and the State Key Program for Basic Research of China (Grant Nos. 2009CB623303 and 2009CB929501).

${ }^{1}$ E. Yablonovitch, Phys. Rev. Lett. 58, 2059 (1987).

${ }^{2}$ S. John, Phys. Rev. Lett. 58, 2486 (1987).

${ }^{3}$ Y. Fink, J. N. Winn, S. Fan, C. Chen, J. Michel, J. D. Joannopoulos, and E. L. Thomas, Science 282, 1679 (1998); S. Fan, P. R. Villeneuve, J. D. Joannopoulos, M. J. Khan, C. Manolatou, and H. A. Haus, Phys. Rev. B 59(15), 882 (1999).

${ }^{4}$ S. G. Johnson, P. R. Villeneuve, S. Fan, and J. D. Joannopoulos, Phys. Rev. B 62, 8212 (2000).

${ }^{5}$ K. Busch and S. John, Phys. Rev. Lett. 85, 967 (1999).

${ }^{6}$ H. Schmid, Ferroelectrics 62, 317 (1994); W. Eerenstein, N. D. Mathur, and J. F. Scott, Nature (London) 442, 756 (2006); W. Prellier, M. P. Singh, and P. Murugavel, J. Phys.: Condens. Matter 17, R803 (2005); K. F. Wang, J. M. Liu, and Z. F. Ren, Adv. Phys. 58(4), 321 (2009).

${ }^{7}$ T. Kimura, T. Goto, H. Shintani, K. Ishizaka, T. Arima, and Y. Tokura, Nature (London) 426, 55 (2003).

${ }^{8}$ N. Hur, S. Park, P. A. Sharma, J. S. Ahn, S. Guha, and S.-W. Cheong, Nature (London) 429, 392 (2004).

${ }^{9}$ A. Pimenov, A. A. Mukhin, V. Yu. Ivanov, V. D. Travkin, A. M. Balbashov, and A. Loidol, Nature Phys. 2, 97 (2006).

${ }^{10}$ K. Sawada and N. Nagaosa, Appl. Phys. Lett. 87, 042503 (2005).

${ }^{11}$ T. Kimura, G. Lawes, T. Goto, Y. Tokura, and A. P. Ramirez, Phys. Rev. B 71, 224425 (2005).

${ }^{12}$ J. Xu, R. X. Wu, P. Chen, and Y. Shi, J. Phys. D 40, 960 (2007); P. Chen, R. X. Wu, J. Xu, A. M. Jiang, and X. Y. Ji, J. Phys.: Condens. Matter 19, 106205 (2007).

${ }^{13}$ D. Senff, P. Link, K. Hradil, A. Hiess, L. P. Regnault, Y. Sidis, N. Aliouane, D. N. Argyriou, and M. Braden, Phys. Rev. Lett. 98, 137206 (2007); D. Senff, N. Aliouane, D. N. Argyriou, A. Hiess, L. P. Regnault, P. Link, K. Hradil, Y. Sidis, and M. Braden, J. Phys.: Condens. Matter 20, 434212 (2008).

${ }^{14}$ C. A. Balanis, Advanced Enginerring Electromechanics (Wiley, New York, 1989).

${ }^{15}$ American translation of "Beiträge zur Optik trüber Medien, speziell kolloidaler Metallösungen” (Mie-SAND78-6018-Mie-1908-translation).

${ }^{16}$ C. F. Bohren and D. R. Huffman, Absorption and Scattering of Light by Small Particles (Wiley, NewYork, 1983). 